\title{
Carbon species confined inside carbon nanotubes: A density functional study
}

\author{
Yi Liu and R. O. Jones* \\ Institut für Festkörperforschung, Forschungszentrum Jülich, D-52425 Jülich, Germany \\ Xinluo Zhao and Yoshinori Ando \\ Department of Materials Science and Engineering, Meijo University, Nagoya 468-8502, Japan
}

(Received 18 March 2003; published 24 September 2003)

\begin{abstract}
Density functional calculations have been performed of the energies, structures, and vibration frequencies of carbon chains, rings, graphitic sheets, bowls, cages, and tubes inside single-walled carbon nanotubes (CNT's) with different diameters. The calculated energies show that carbon chains can be inserted coaxially into $0.7 \mathrm{~nm}$ nanotubes, and nanotubes with diameters larger than $1.0 \mathrm{~nm}$ can also accommodate other structures. Small carbon species without dangling bonds do not touch the CNT walls, but those with dangling bonds can react with wall atoms, leading to structures with mixed hybridization $\left(s p, s p^{2}\right.$, and $\left.s p^{3}\right)$. Carbon chains and rings have stretching modes between 1800 and $2160 \mathrm{~cm}^{-1}$, and chain modes are softened when inserted into CNT's. Normal modes of graphitic sheets and cages lie below $1645 \mathrm{~cm}^{-1}$. The calculations suggest the existence of carbon nanowires, and nanopeapods $\mathrm{C}_{20} @ \mathrm{CNT}$ should form if the CNT diameter is more than $1 \mathrm{~nm}$. The smallest CNT $(2,2)$ [diameter $0.3 \mathrm{~nm}$ ] should be stable as the innermost tube of multiwalled nanotubes.
\end{abstract}

DOI: 10.1103/PhysRevB.68.125413

PACS number(s): 78.67.Ch, 81.07.De, 71.15.Mb

\section{INTRODUCTION}

The identification of the cagelike structure of $\mathrm{C}_{60}$ (Ref. 1) led to a surge of interest in carbon molecules, and an additional impetus came in 1991 with the discovery of carbon nanotubes (CNT's). ${ }^{2}$ The properties of these hollow cylindrical molecules are remarkably dependent on their diameter and helicity, and inserting other molecules into them has potential applications as molecular sieves, nanoreactors, and nanocontainers. ${ }^{3}$

Nanotubes often form as multiwalled nanotubes (MWNT's), ${ }^{2}$ and there has been much speculation about the mechanism of their formation. It has been proposed that a single-walled nanotube (SWNT) forms from a fullerene "dome" by growing inwards and outwards into a MWNT., During inward growth, carbon atoms enter the core to form thinner CNT's, a process that continues until prevented by the narrowness of the innermost tube. Carbon atoms can be trapped inside the cores during such a process, and confinement might allow the preparation of long chains or other molecules that are not stable in the gas phase. Linear carbon chains with $s p$ hybridization account for many features of diffuse interstellar bands ${ }^{6}$ and are examples of carbon molecules that have been studied for many years. ${ }^{7}$ Such chains are, however, highly reactive and difficult to isolate and identify in the gas phase.

The CNT's considered here are usually less than $1 \mathrm{~nm}$ in diameter. Although it is difficult to characterize structures on this scale, recent experimental progress is very encouraging. High-resolution transmission electron microscopy (HRTEM) measurements and Raman scattering studies indicate that long carbon chains can be inserted into the core of MWNT's. ${ }^{8}$ HRTEM also suggests that a CNT of diameter $0.3 \mathrm{~nm}$ can be prepared as the innermost tube of a MWNT. ${ }^{9}$ The record for the narrowest CNT has been lowered continuously since their first discovery: from $0.8 \mathrm{~nm}$ (Ref. 10), 0.5 $\mathrm{nm}$ (Ref. 11), and $0.4 \mathrm{~nm}$ (Ref. 12) to $0.34 \mathrm{~nm}$ (Ref. 13) and
$0.33 \mathrm{~nm}$ (Ref. 14). The determination of the smallest stable CNT, whether isolated or confined in a MWNT, is a continuing challenge to theory. ${ }^{14-18}$

We study here the structures and properties of carbon species confined in small CNT's using a combined density functional (DF) and molecular dynamics (MD) method. ${ }^{19} \mathrm{We}$ have investigated the energies, geometries, and vibrational properties of carbon chains, rings, graphitic sheets, bowls, cages, and tubes inside CNT's from $(4,4)$ (diameter 0.553 $\mathrm{nm})$ to $(7,7)$ (diameter $0.953 \mathrm{~nm}$ ). The numerical method used is described in Sec. II, and we present and discuss the results in Secs. III and IV, respectively. Our concluding remarks follow in Sec. V.

\section{COMPUTATIONAL METHOD}

We have used DF calculations with simulated annealing. ${ }^{19}$ The electron-ion interaction is described by ionic pseudopotentials of the form of Troullier and Martins, ${ }^{20}$ and the exchange-correlation energy uses the approximation of Perdew, Burke, and Ernzerhof (PBE). ${ }^{21}$ We use periodic boundary conditions with a single point $(\vec{k}=0)$ in the Brillouin zone, and the wave functions are expanded in a plane-wave basis with a kinetic energy cutoff of $50 \mathrm{Ry}$.

An orthorhombic unit cell was used in all calculations, and the separation between CNT is at least $0.8 \mathrm{~nm}$. The supercell models of the CNT include five unit cells: CNT $(2,2)$ (40 atoms), (4,4) (80 atoms), (5,5) (100 atoms), (6,6) (120 atoms), and (7,7) (140 atoms). The supercells along the CNT axis are about $1.24 \mathrm{~nm}$ long, and typical cells are shown in Figs. 1-3. The confined carbon species include chains, rings, graphitic sheets, bowls, cages, and tubes. The geometries have been optimized using simulated annealing. ${ }^{19}$ The vibration frequencies and eigenvectors were calculated using finite differences with atomic displacements of $0.001 \mathrm{~nm}$. 
(a)

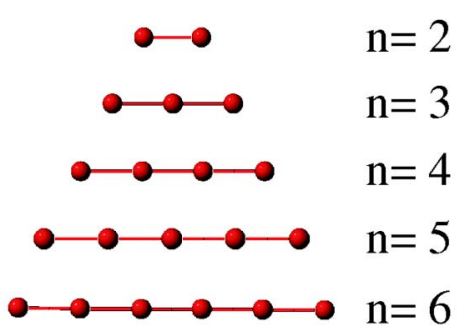

(b)

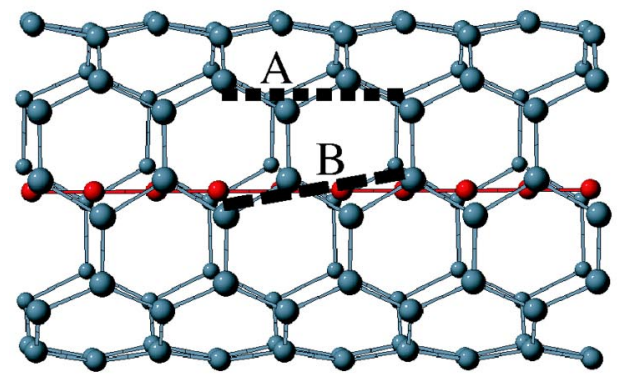

(c)

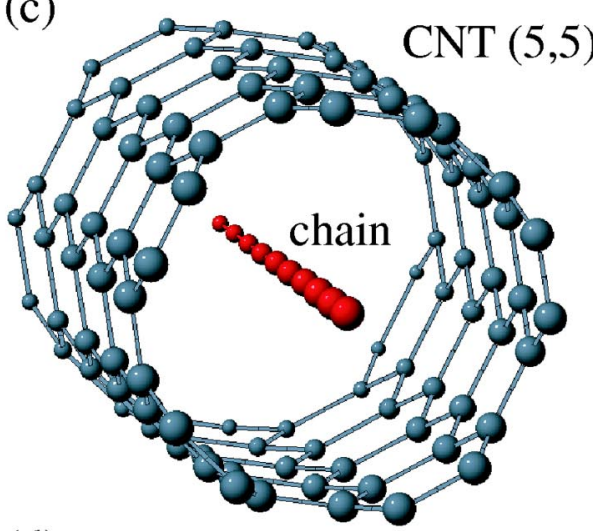

(d)

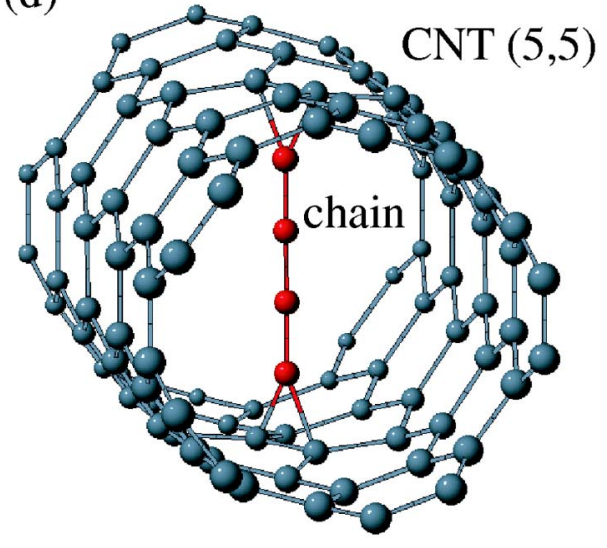

FIG. 1. (Color online) Supercell models of carbon chains inside CNT. (a) Carbon chains $\mathrm{C}_{n}(n=2-6)$, (b) side view of chain10@(5,5). The dotted line A shows the parallel off-center position and the dashed line $\mathrm{B}$ represents the inclined position described in text, (c) top view of chain10@(5,5), (d) chain4@ $(5,5)$, with a carbon chain perpendicular to the nanotube axis.

\section{CARBON SPECIES INSIDE NANOTUBES}

The relative stabilities of carbon species are determined by the binding energies, which are defined as the differences

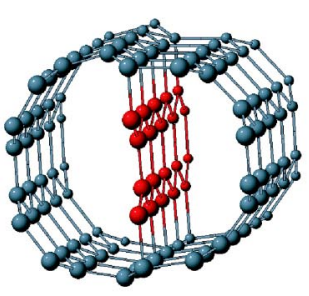

(a) sheet20@ $(5,5)$

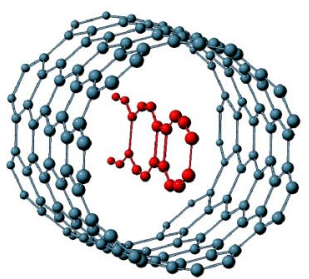

(c) sheet20@(7,7)

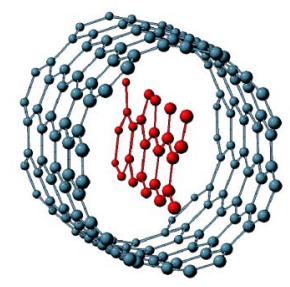

(e) sheet25@(7,7)

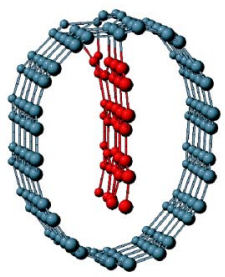

(g) sheet35@(7,7)
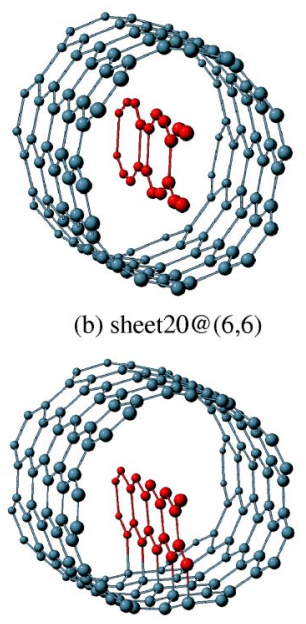

(d) sheet20@(7,7) (off-center)

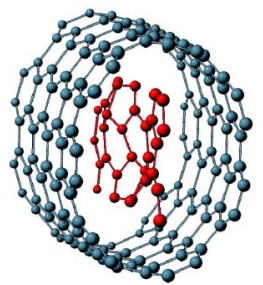

(f) sheet30@(7,7)

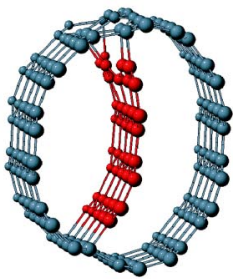

(h) sheet40@(7,7) (b) sheet $20 @(6,6)$

FIG. 2. (Color online) Supercell models of (a), (d)-(h) graphitic sheets and (b), (c) carbon rings inside CNT's.

of total energies before and after carbon species (csp) are inserted into the CNT, i.e.,

$$
E_{\text {bind }}=\left[E_{t o t}(\mathrm{csp})+E_{t o t}(\mathrm{CNT})\right]-E_{t o t}(\mathrm{csp} @ \mathrm{CNT}) .
$$

$E_{t o t}(\mathrm{csp})$ and $E_{t o t}(\mathrm{CNT})$ are the total energies of isolated carbon species and CNT, respectively, and $E_{\text {tot }}(\mathrm{csp} @ \mathrm{CNT})$ is the total energy of the CNT with the additional carbon species. A large $E_{\text {bind }}$ means strong binding to the nanotube. The armchair CNT $(2,2),(4,4),(5,5),(6,6)$, and $(7,7)$ studied here have optimized diameters of $0.281 \mathrm{~nm}, 0.553 \mathrm{~nm}, 0.684$ $\mathrm{nm}, 0.822 \mathrm{~nm}$, and $0.953 \mathrm{~nm}$, respectively. All extended systems are calculated as singlet states. For chains with fewer than ten atoms, the most stable form is a triplet (singlet) if the number of atoms is even (odd), and we use the lowest energy for $E_{t o t}(\mathrm{csp})$ in evaluating $E_{\text {bind }}$ from Eq. (1).

\section{A. Carbon chains}

The effect of the length of linear carbon chains ( $n$ $=2-6)$ [Fig. 1(a)] on the binding energy is shown for CNT $(5,5)$ in Fig. 4. The minimum separation between the chains is about $0.6 \mathrm{~nm}$ for $n=6$, so the interactions between the 


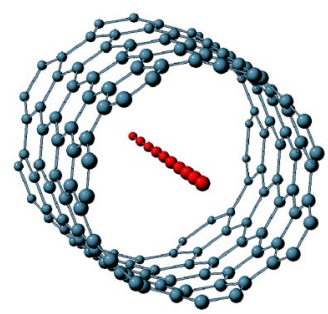

(a) chain@(7,7)
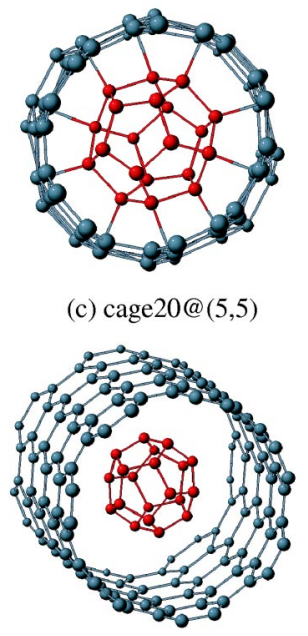

(e) cage20@(7,7) (c) cage20@(5,5)

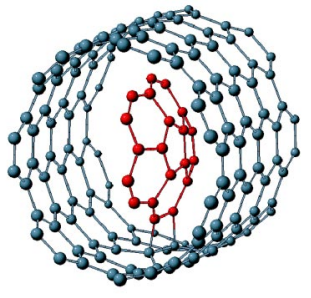

(b) bow120@(7,7)

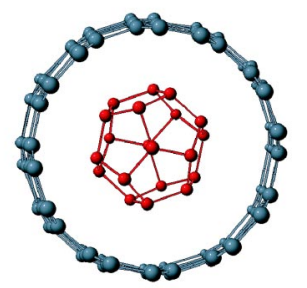

(d) cage20@(6,6)

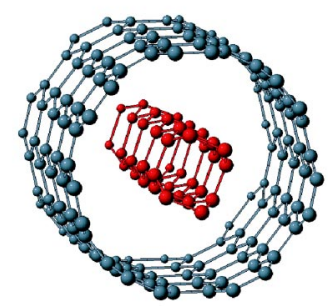

(f) $(2,2) @(7,7)$
FIG. 3. (Color online) Supercell models of (a) carbon chain, (b) bowl, (c)-(e) cages, (f) tube inside CNT's.

chain images are negligible. The geometry of a four-atom chain inside $(5,5)$ [denoted chain4@ $(5,5)$ ] was optimized for several initial geometries, and the most stable (with binding energy $-0.07 \mathrm{eV}$ ) is a chain along the axis of the CNT. Other starting configurations, such as an off-center position parallel to the axis [dotted in Fig. 1(b)] and a tilted chain [dashed in Fig. 1(b)], also led to the coaxial geometry. If the initial chain geometry is perpendicular to the CNT axis [Fig. $1(\mathrm{~d})$ ], there is relatively little relaxation to the next energy minimum, but the structure is not bound (with binding energy $-1.95 \mathrm{eV})$. The triangular configurations needed to connect the chain and CNT wall are clearly unfavorable, and some atoms appear to be removed from the CNT wall. Chains from chain2 to chain6 are weakly bound or slightly unbound when inserted along the axis of CNT $(5,5)$, as shown in Fig. 4.

The four-atom chain has been inserted into CNT's from $(4,4)$ to $(7,7)$ [with diameters from $0.55 \mathrm{~nm}$ to $0.95 \mathrm{~nm}$ ], and the binding energies are shown in Fig. 4. The binding energy of chain4 inside nanotube $(4,4)$ is large and negative, indicating that the diameter of $\mathrm{CNT}(4,4)$ is too small to accommodate linear chains. The four-membered chain is weakly bound for CNT $(6,6)$, but it is slightly unbound in $\operatorname{CNT}(5,5)$ and $(7,7)$. The binding energy should approach zero for very large CNT diameters, where there is little interaction between chains and nanotubes. The diameter of CNT $(5,5)$ $(0.684 \mathrm{~nm})$ is unchanged when the carbon chain is inserted. The distance between coaxial chains and nanotube wall is $0.342 \mathrm{~nm}$, in good agreement with the interlayer distance in

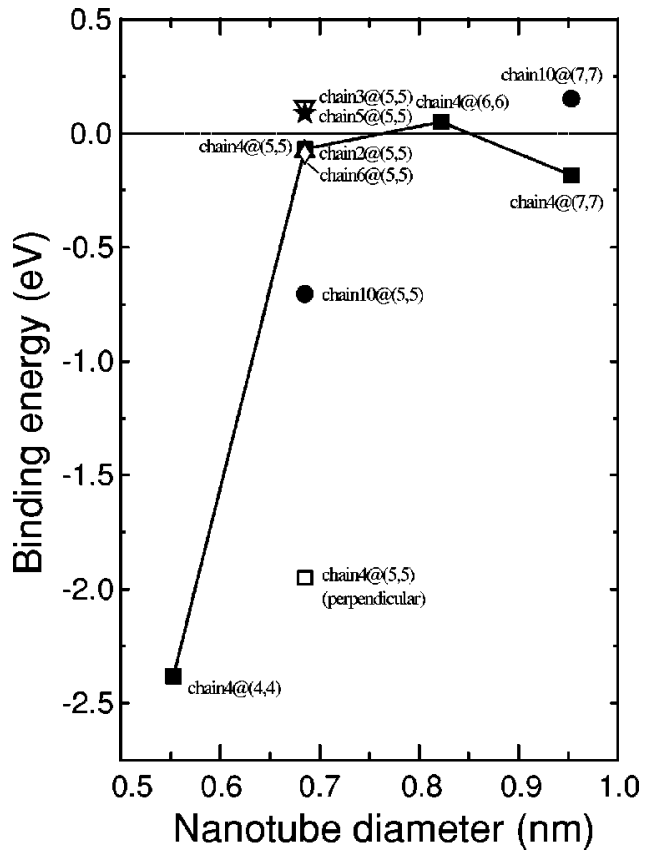

FIG. 4. Dependence of binding energies on nanotube diameter for carbon chains inside CNT's.

graphite $(0.34 \mathrm{~nm})$ and the recent HRTEM observation, ${ }^{8}$ which shows the innermost tubes with radius $0.35 \mathrm{~nm}$ containing carbon chains.

To simulate an infinite carbon chain, a ten-atom chain was inserted into nanotubes $(5,5)$ and $(7,7)$ [see Figs. 1(b), 1(c), and 3(a)]. Figure 4 shows that the binding energy is negative in the former case, but positive in $(7,7)$. The results of the calculations for the ten-atom chains cannot be compared directly with those for the shorter chains, since $C_{10}$ is forced to be commensurate with the supercell, and relaxation of this restriction would result in stronger binding. The fully relaxed $\mathrm{C}-\mathrm{C}$ bonds in chain4 inside $\mathrm{CNT}(4,4)$ are $0.129 \mathrm{~nm}, 0.134$ $\mathrm{nm}$, and $0.129 \mathrm{~nm}$; i.e., they are modified cumulenic bonds as found in $\mathrm{C}_{10}$.

\section{B. Carbon rings, graphitic sheets, and bowls}

Two-dimensional graphitic sheets (Fig. 2) containing 20-40 atoms per supercell (denoted sheet20 to sheet40) were inserted into CNT's and their structures optimized. The initial geometry of sheet20, a strip of connected hexagons, changed little on insertion into CNT $(5,5)$ [Fig. 2(a)]. The dangling bonds of the edge atoms are eliminated by bonding to the CNT wall, atoms of which move towards the sheet to form a double-tube or tube-sheet hybrid structure. Figure 2(b) shows that placing sheet20 in the center of CNT $(6,6)$ results in the conversion of six-membered rings to connected rings with a mixture of eight-membered rings and squares.

The structure of the connected hexagons was also optimized without the outer CNT. As in the confined geometry, the initial six-membered rings changed to connected 12membered rings and squares. The geometry changes can be viewed as ways to release strain, and similar structures have been found for isolated carbon clusters. ${ }^{7}$ On the other hand, 


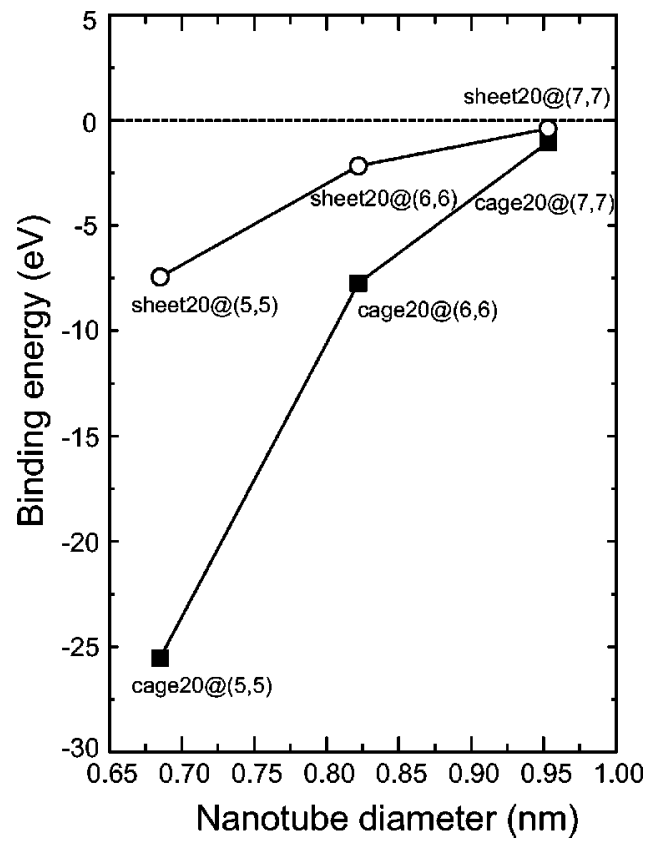

FIG. 5. Dependence of binding energies on nanotube diameter for graphitic sheets/carbon rings and cages inside CNT's.

the radial deformation of the CNT points to a repulsive interaction between sheet20 and $(6,6)$. The hexagons inserted into the center of CNT $(7,7)$ relax in a similar fashion to that in $(6,6)$, with less deformation of the CNT [Fig. 2(c)]. The hexagons were also put close to the $(7,7)$ tube wall, an offcenter position shown in Fig. 2(d), and relaxation led to pinning of the six-membered rings to the CNT wall. This is similar to those inside $(5,5)$, except that only one side bonds to the wall.

Insertion of sheet20 into CNT $(5,5)$ and $(7,7)$ does not lead to binding (Fig. 5), but $E_{\text {bind }}$ increases gradually as the nanotubes become larger, reaching $-0.39 \mathrm{eV}$ for $(7,7)$ (diameter $0.953 \mathrm{~nm})$. The off-center sheet20@(7,7) structure [Fig. 2(d)] is much less stable, with a binding energy of $-7.23 \mathrm{eV}$.

Narrow strips of graphitic layers were inserted into CNT $(7,7)$, as shown in Figs. 2(e)-2(h). When the sheets are small compared with the outer nanotube [Figs. 2(e) and 2(f)], the atoms at the edge of sheets tend to form pentagons and nonplanar structures, thereby eliminating the dangling bonds at the edges. When the sheet size increases, wall atoms can be displaced on forming bonds to edge atoms of the sheet [Figs. $2(\mathrm{~g})$ and $2(\mathrm{~h})]$, increasing both nanotube deformation and sheet bending. The binding energies of these graphitic sheets included in CNT are $-1.85 \mathrm{eV}$ [sheet25@(7,7)], $1.52 \mathrm{eV}$ [sheet30@(7,7)], - $1.12 \mathrm{eV}[$ sheet35@(7,7)], and $2.02 \mathrm{eV}$ [sheet40@(7,7)], respectively. The last of these is the most stable, but the original tube structure is very distorted. Sheet40 is unlikely to be stable inside the stabilized $(7,7)$ nanotube expected in a MWNT (see below).

The carbon bowl can be obtained from a graphitic sheet by replacing a hexagon by a pentagon. As shown in Fig. 3 (b), bowl20 is pinned to the wall of CNT $(7,7)$ through bonding between bowl edge atoms and tube wall atoms. The insertion of bowl20 into $(7,7)$ leads to deformation of the
CNT and a negative binding energy of $-3.40 \mathrm{eV}$. Sheet or bowl insertion is favored by rearrangement to saturate dangling bonds or by bonding with the CNT wall, but is hindered by the deformation of sheets, bowls, and nanotubes.

\section{Carbon cages and tubes}

The smallest fullerene cage20 (comprising 12 pentagons) has been studied experimentally ${ }^{22}$ and theoretically. ${ }^{23}$ As shown in Figs. 3(c)-3(e), cage20 has been inserted into the center of CNT $(5,5),(6,6)$, and $(7,7)$, respectively. Insertion into $(5,5)$ results in radial expansion of the CNT [Fig. 3(c)] and a very unfavorable binding energy $(-25.54 \mathrm{eV})$. The radial expansions [Fig. 3(c)-3(e)] are lowered, and the binding energies (Fig. 5) become much more favorable $[-1.03 \mathrm{eV}$ for $(7,7)$ with diameter $0.953 \mathrm{~nm}]$ as the nanotube diameter increases. The minimum interatomic distance between cage 20 and CNT $(7,7)$ is about $0.283 \mathrm{~nm}$, less than the interlayer separation in graphite. Figure 5 shows that the binding energies of both cage 20 and sheet 20 approach zero as the CNT diameter increases. These results show that nanotubes larger than $(7,7)$ would be needed to accommodate cage 20 as a nanopeapod. ${ }^{24}$ If cage 20 is assumed to have an initial off-center position close to the wall of CNT $(7,7)$, it reverts to the central position inside the CNT.

Figure 3(f) shows the double-walled nanotube (DWNT) $(2,2) @(7,7)$. The optimized diameter of CNT $(2,2)$ in this case is $0.30 \mathrm{~nm}$, which is larger than that of a rolled graphitic sheet. The minimum interatomic distance between $(2,2)$ and $(7,7)$ is about $0.336 \mathrm{~nm}$, close to the interlayer distance in graphite $(0.34 \mathrm{~nm})$, and the calculated geometries are consistent with HRTEM measurements. ${ }^{9}$ The binding energy of CNT $(2,2) @(7,7)$ is $-0.90 \mathrm{eV}$, and unrolling CNT $(2,2)$ to a graphitic sheet (sheet40) lowers the energy by $0.27 \mathrm{eV}$ per atom. After insertion into CNT $(7,7)$, the total energy per atom of the sheet is $0.08 \mathrm{eV}$ lower than that of CNT $(2,2)$. Both the graphitic sheet and CNT deform when sheet40 is inserted into $(7,7)$ [Fig. 2(h)]. The innermost nanotube deforms little in MWNT's, and the total energy of sheet40@MWNT should increase because of additional confinement. These results show that outer nanotubes may impose restrictions on the formation of graphitic sheet, so that it is more favorable to form CNT $(2,2)$ in the core of MWNT than as a SWNT $(2,2)$.

\section{Vibration frequencies}

Vibration frequencies have been calculated for all structures. The frequency for the Raman active breathing mode (BM) of SWNT $(5,5)\left(370 \mathrm{~cm}^{-1}\right)$ is close to the tightbinding value for $\operatorname{SWNT}(6,4)\left(366 \mathrm{~cm}^{-1}\right),{ }^{25}$ which has a similar diameter $(0.69 \mathrm{~nm})$. The BM frequency can be estimated from the inverse function obtained from force constant calculations: ${ }^{26}$

$$
\omega=\frac{223.75}{d} \mathrm{~cm}^{-1}
$$

where $d$ is the CNT diameter in $\mathrm{nm}$. This formula predicts a frequency for SWNT $(5,5)$ of $327 \mathrm{~cm}^{-1}$. The deviation of 


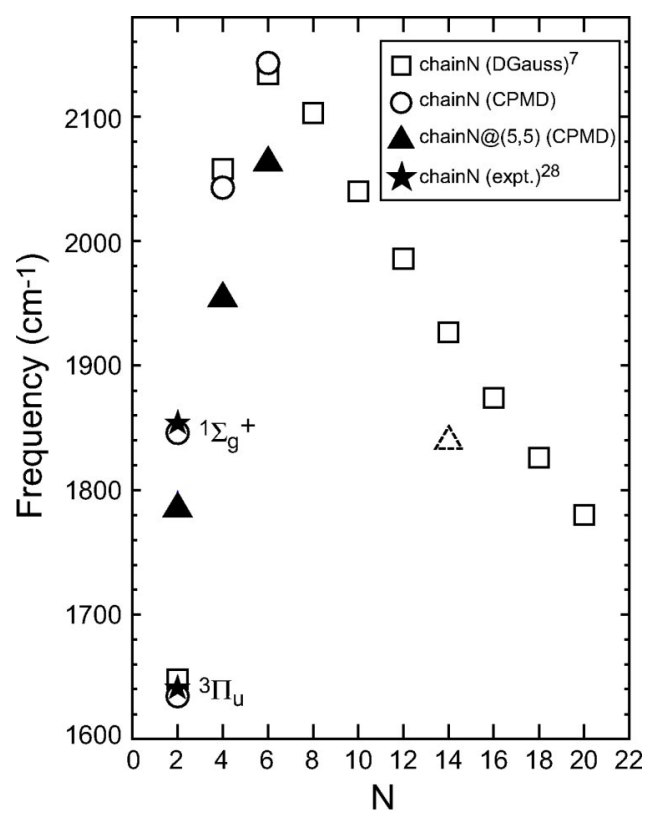

FIG. 6. Frequency of the stretching mode of carbon chains as a function of the number of carbon atoms.

calculated BM from the simple inverse function is also evident in other DF calculations for small CNT. ${ }^{27}$ The BM of chain4@(5,5) is $374 \mathrm{~cm}^{-1}$, indicating that chain insertion changes its value little. Our calculated value of the BM of SWNT $(2,2)\left(787 \mathrm{~cm}^{-1}\right)$ is closer to the estimate from Eq. (2) $\left(796 \mathrm{~cm}^{-1}\right)$. The vibrational analysis of $\operatorname{DWNT}(2,2) @(7,7)$ shows that the BM of $(2,2)$ is $787 \mathrm{~cm}^{-1}$, indicating that the outer nanotube has little effect on the BM frequency of the inner CNT.

Linear chains have a center of symmetry for all $n$, so that Raman-active modes are totally symmetrical. The frequencies of bending modes $\left(50-500 \mathrm{~cm}^{-1}\right)$ are very low, and we focus on the $\Sigma_{g}$ stretching mode, where neighboring carbon atoms vibrate out of phase. The dependence of vibration frequency on the (even) number of carbon atoms in the chain is shown in Fig. 6. The open squares in Fig. 6 are the frequencies of isolated chains $\mathrm{C}_{n}(n=2-20)$ calculated with an allelectron, Gaussian basis DF method. ${ }^{7}$ The frequency increases as the number of carbon atoms $n$ increases to a maximum at $n=6$, after which it decreases. The open circles and solid triangles in Fig. 6 show the frequencies of chain $(n=2-6)$ calculated without and with CNT $(5,5)$. The experimental values are shown if available. ${ }^{28}$ The vibration frequencies for chains inserted into $(5,5)$ are lower by about $60-90 \mathrm{~cm}^{-1}$ than those of isolated chains.

Recent Raman scattering experiments identified a strong peak around $1850 \mathrm{~cm}^{-1}$ for a MWNT containing carbon chains, ${ }^{8}$ and the corresponding HRTEM images indicate that the chains can be as long as $20 \mathrm{~nm}$. The chains might comprise shorter segments separated by vacancies, and this suggests a possible origin of the peak in the Raman spectrum. We have noted that the vibration frequencies of chains inserted into the CNT $(5,5)$ are lower than those of the isolated chains, so that the frequency of an inserted chain with about 14 atoms leads to a frequency of approximately $1850 \mathrm{~cm}^{-1}$ (the open dotted triangle in Fig. 6). The stretching mode for chain4@(5,5) has a frequency of $1954 \mathrm{~cm}^{-1}$, increasing to $1998 \mathrm{~cm}^{-1}$ for chain4@(6,6) and $1993 \mathrm{~cm}^{-1}$ for chain4@(7,7). These results suggest that vibration frequencies of carbon chains increase upon insertion into larger CNT, approaching a value of about $2000 \mathrm{~cm}^{-1}$.

Vibration frequencies have also been calculated for carbon rings, graphitic sheets, cages, and tubes. The stretching modes of connected rings in sheet20@(7,7) [Fig. 2(c)] give a maximum vibration frequency up to $2155 \mathrm{~cm}^{-1}$. The calculations for sheet20@(5,5) [Fig. 2(a)] and sheet20@(7,7) in the case of off-center position [Fig. 2(d)] show that all vibration frequencies of sheets are lower than $1645 \mathrm{~cm}^{-1}$. The cage inserted into $(7,7)$ [Fig. 3(e)] has vibration frequencies no higher than $1450 \mathrm{~cm}^{-1}$, and the maximum vibration frequency of $\mathrm{CNT}(2,2)$ inside $(7,7)$ [Fig. 3(f)] is $1600 \mathrm{~cm}^{-1}$.

\section{DISCUSSION}

We have studied the structures and energetics of a variety of carbon systems inside carbon nanotubes, and we now focus on the trends that are found. The diameter of the nanotube plays a central role in all cases. We consider singlet states of the CNT with its enclosed species (csp), and the interaction between CNT and csp will be repulsive if the CNT diameter is small. This is evident in chain4@ CNT $(4,4)$, and the repulsion is reduced as the CNT diameter increases. For very large diameters the interaction (and the binding energy) should be small, and this is evident in both chains (Fig. 4) and for 20-atom sheets and cages (Fig. 5). The cross-bonded four-atom chains lead to a distortion of the CNT and an unfavorable energy. Figure 4 shows that the binding energy of chain4 to CNT depends on the nanotube diameter, being greatest in $\operatorname{CNT}(6,6)$. The continuous chain10@CNT $(7,7)$ has the same number of bonds and atoms, whereas chain4@ CNT $(7,7)$ has one bond fewer than the number of atoms, so that it is plausible that the former has a larger binding energy.

In all cases where we have started the calculations with the chain in an off-axial structure, energy optimization has resulted in an axial structure. We note that the CNT play an essential role in stabilizing the chain structures, since almost all isolated chains have bending modes with imaginary frequencies. Differences between an ideal one-dimensional system and the same system inside a CNT have also been noted in the case of diffusion Monte Carlo calculations of ${ }^{4} \mathrm{He}$ inside a narrow single-walled $\mathrm{CNT}^{29}$

Insertion of carbon chains into CNT $(5,5)$ (diameter 0.684 $\mathrm{nm}$ ) is energetically more favorable than for sheets or cages. Although many of the structures we consider are unbound in the sense of Eq. (1), the variation of the binding energies as the CNT diameter changes indicates that CNT larger than $(7,7)$ can include carbon chains and larger carbon species. For example, the connected carbon rings [Fig. 2(c)], cage20 [Fig. 3(e)] and nanotube (2,2) [Fig. 3(f)] have small binding energies in CNT $(7,7)$. Insertion into CNT $(7,7)$ is favored increasingly in the order chains, connecting rings, $\operatorname{CNT}(2,2)$ and cage 20 .

The bonding characteristics of many structures are inter- 
esting. Figures 1(d) and 2(a), 2(d), 2(g), and 2(h) show that the two-, three-, and four-coordinated $\mathrm{C}$ atoms can coexist in chain-tube or sheet-tube hybrid structures corresponding to $s p, s p^{2}$, and $s p^{3}$ hybridization. In addition to confinement, CNT's can provide atoms to interact with other carbon species. Atoms can accumulate at the CNT wall, forming new molecules or clusters with mixed hybridized bonding.

\section{CONCLUDING REMARKS}

We have performed density functional calculations of the energies, geometries, and vibration frequencies of carbon chains, rings, graphitic sheets, bowls, cages, and tubes inside single-walled nanotubes. The armchair nanotubes studied $[(n, n), n=4-7]$ have diameters between $0.553 \mathrm{~nm}$ and $0.953 \mathrm{~nm}$. CNT's with diameters above $0.7 \mathrm{~nm}$ can accommodate linear carbon chains, and those with diameters larger than $1.0 \mathrm{~nm}$ can also incorporate rings, graphitic sheets, smaller nanotubes, or cages. Species with dangling bonds can interact with the CNT wall or form other stable structures, depending on the distance from the CNT wall. For carbon species with saturated bonds, the minimum distance from the CNT wall (about $0.34 \mathrm{~nm}$ ) is close to the interlayer spacing in graphite. The large carbon species can induce both local and long-range deformations of CNT.

The present discussion has focused on the total energy. Some of the energetically unfavorable structures could, however, occur under conditions far from equilibrium, such as the extremely high temperatures used to generate CNT. On the other hand, the existence of structures with low-lying energies does not guarantee that they will form.

The frequencies of the stretching modes of carbon chains and rings are above $1800 \mathrm{~cm}^{-1}$, those of graphitic sheets are below $1645 \mathrm{~cm}^{-1}$, and the maximum frequencies of cages are $1450 \mathrm{~cm}^{-1}$. The frequency of stretching modes of carbon chains is reduced on insertion into CNT, so that the Raman peak observed at $1850 \mathrm{~cm}^{-1}$ (Ref. 8) is probably due to carbon chains, since carbon rings are too large to form inside CNT $(5,5)$. Isolated carbon rings $\left(\mathrm{C}_{18}\right.$ and $\left.\mathrm{C}_{20}\right)$ (Ref. 7) have Raman-active modes around $2100 \mathrm{~cm}^{-1}$, but none in the range $1700-2000 \mathrm{~cm}^{-1}$. Recently Ravagnan et al. ${ }^{30}$ assigned to carbon chains a Raman peak found near $2100 \mathrm{~cm}^{-1}$ in deposited amorphous carbon films. Such frequencies also occur in the vibrations of rings, which are much more likely in films than in the confined geometries of CNT's. We also note that isolated rings are more stable than isolated chains for $n>10 .^{7}$

Our calculations support the existence of carbon nanowires (CNW's), which have been found recently in MWNT's, ${ }^{8}$ and they suggest that nanotube confinement in a MWNT could stabilize the smallest CNT $(2,2)$ with diameter $0.3 \mathrm{~nm} .{ }^{9}$ These novel carbon structures present an exciting field for further investigation.

\section{ACKNOWLEDGMENTS}

This work has been supported by the Bundesministerium für Bildung und Forschung, Bonn, Germany (Kompetenzzentrum Werkstoffmodellierung, Grant No. 03N6015). We thank the Forschungszentrum Jülich, Germany, and the John von Neumann Institute for Computing (NIC) for generous access to the Cray T3E supercomputers in the FZ Jülich, and the Research Center for Computational Science (RCCS) at the Okazaki National Research Institute, Japan, for a grant of time on the Fujitsu VPP5000 supercomputers. We thank P. Milani for providing information (Ref. 30) prior to publication.
*Corresponding author. Electronic address: r.jones@fz-juelich.de

${ }^{1}$ H.W. Kroto, J.R. Heath, S.C. O'Brien, R.F. Curl, and R.E. Smalley, Nature (London) 318, 162 (1985).

${ }^{2}$ S. Iijima, Nature (London) 354, 56 (1991).

${ }^{3}$ See, for example, B. Ni, S.B. Sinnott, P.T. Mikulski, and J.A. Harrison, Phys. Rev. Lett. 88, 205505 (2002), and references therein.

${ }^{4}$ S. Iijima, P.M. Ajayan, and T. Ichihashi, Phys. Rev. Lett. 69, 3100 (1992)

${ }^{5}$ S. Amelinckx, D. Bernaerts, X.B. Zhang, G. Van Tenderloo, and J. Van Landuyt, Science 67, 1334 (1995).

${ }^{6}$ W.W. Duley, Astrophys. J. 89, 285506 (2000).

${ }^{7}$ R.O. Jones, J. Chem. Phys. 110, 5189 (1999), and references therein. The vibration frequencies of the linear chains have not been published before.

${ }^{8}$ X. Zhao, Y. Ando, Y. Liu, M. Jinno, and T. Suzuki, Phys. Rev. Lett. 90, 187401 (2003).

${ }^{9}$ X. Zhao, Y. Liu, S. Inoue, M. Jinno, T. Suzuki, R. O. Jones, and Y. Ando (unpublished).

${ }^{10}$ P.M. Ajayan and S. Iijima, Nature (London) 358, 23 (1992).

${ }^{11}$ L.F. Sun, S.S. Xie, W. Liu, W.Y. Zhou, Z.Q. Liu, D.S. Tang, G. Wang, and L.X. Qian, Nature (London) 403, 384 (2000).

${ }^{12}$ L.C. Qin, X. Zhao, K. Hirahara, Y. Miyamoto, Y. Ando, and S.
Iijima, Nature (London) 408, 50 (2000); N. Wang, Z.K. Tang, G.D. Li, and J.S. Chen, ibid. 408, 50 (2000).

${ }^{13}$ Y. Li, S. Xie, W. Zhou, D. Tang, Z. Liu, X. Zou, and G. Wang, Carbon 39, 1429 (2001).

${ }^{14}$ L.M. Peng, Z.L. Zhang, Z.Q. Xue, Q.D. Wu, Z.N. Gu, and D.G. Pettifor, Phys. Rev. Lett. 85, 3249 (2000).

${ }^{15}$ D.H. Robertson, D.W. Brenner, and J.W. Mintmire, Phys. Rev. B 45, 12592 (1992).

${ }^{16}$ S. Sawada and H. Hamada, Solid State Commun. 83, 917 (1992).

${ }^{17}$ A.A. Lucas, P.H. Lambin, and R.E. Smalley, J. Phys. Chem. Solids 54, 587 (1993).

${ }^{18}$ X. Blase, L.X. Benedict, E.L. Shirley, and S.G. Louie, Phys. Rev. Lett. 72, 1878 (1994).

${ }^{19}$ J. Hutter et al., Computer Code CPMD, version 3.5. IBM Corp, 1990-2001, and MPI für Festkörperforschung, Stuttgart, Germany, 1997-2001.

${ }^{20}$ N. Troullier and J.L. Martins, Phys. Rev. B 43, 1993 (1991).

${ }^{21}$ J.P. Perdew, K. Burke, and M. Ernzerhof, Phys. Rev. Lett. 77, 3865 (1996).

${ }^{22}$ H. Prinzbach, A. Weiler, P. Landenberger, F. Wahl, J. Wörth, L.T. Scott, M. Gelmont, D. Olevano, and B. v. Issendorff, Nature (London) 407, 60 (2000).

${ }^{23}$ M. Saito and Y. Miyamoto, Phys. Rev. Lett. 87, 035503 (2001). 
${ }^{24}$ B.W. Smith, M. Monthioux, and D.E. Luzzi, Nature (London) 396, 323 (1998).

${ }^{25}$ L. Henrard, E. Hernandez, P. Bernier, and A. Rubio, Phys. Rev. B 60, 8521 (1999).

${ }^{26}$ S. Bandow, S. Asaka, Y. Saito, A.M. Rao, L. Grigorian, E. Richter, and P.C. Eklund, Phys. Rev. Lett. 80, 3779 (1998).

${ }^{27}$ D. Sánchez-Portal, E. Artacho, J.M. Soler, A. Rubio, and P. Ordejón, Phys. Rev. B 59, 12678 (1999).
${ }^{28}$ K. P. Huber and G. Herzberg, Molecular Spectra and Molecular Structure. IV. Constants of Diatomic Molecules (Van Nostrand Reinhold, New York, 1979).

${ }^{29}$ M.C. Gordillo, J. Boronat, and J. Casulleras, Phys. Rev. B 61, R878 (2000).

${ }^{30}$ L. Ravagnan, F. Siviero, C. Lenardi, P. Piseri, E. Barborini, P. Milani, C.S. Casari, A. Li Bassi, and C.E. Bottani, Phys. Rev. Lett. 89, 285506 (2002). 